This item was submitted to Loughborough's Research Repository by the author.

Items in Figshare are protected by copyright, with all rights reserved, unless otherwise indicated.

\title{
Using the vibration envelope as a damage-sensitive feature in composite beam structures
}

PLEASE CITE THE PUBLISHED VERSION

http://dx.doi.org/10.1016/j.istruc.2014.10.001

\section{PUBLISHER}

Elsevier Ltd / @ The Institution of Structural Engineers

VERSION

AM (Accepted Manuscript)

\section{PUBLISHER STATEMENT}

This work is made available according to the conditions of the Creative Commons Attribution-NonCommercialNoDerivatives 4.0 International (CC BY-NC-ND 4.0) licence. Full details of this licence are available at: https://creativecommons.org/licenses/by-nc-nd/4.0/

\section{LICENCE}

CC BY-NC-ND 4.0

\section{REPOSITORY RECORD}

Kasinos, Stavros, Alessandro Palmeri, and Mariateresa Lombardo. 2014. "Using the Vibration Envelope as a Damage-sensitive Feature in Composite Beam Structures”. Loughborough University.

https://hdl.handle.net/2134/17376. 


\title{
Using the vibration envelope as damage-sensitive feature in composite beam structures
}

\author{
Stavros Kasinos ${ }^{\mathrm{a}}$, Alessandro Palmeria, ${ }^{\mathrm{a}, *}$, Mariateresa Lombardo ${ }^{\mathrm{a}}$ \\ ${ }^{a}$ School of Civil and Building Engineering, Loughborough University, Sir Frank Gibb \\ Building, Loughborough LE11 3TU, United Kingdom
}

\begin{abstract}
A novel approach of damage detection in composite steel-concrete composite beams is suggested. Based on the idea of using the envelope's profile deflections and rotations induced by a moving load, this approach can lead to a practical cost-effective alternative to the traditional use of accelerometers and laser vibrometers. A parametric study has been undertaken, quantifying the sensitivity of the dynamic response of a realistic composite bridge to the presence of damage at different levels of partial steel-concrete interaction and velocity of the moving load. When compared to shifts in the natural frequencies, it has been verified that the proposed approach generally enjoys a higher sensitivity (so damage can be detected at an early stage), is more effective closer to the ends of the bridge (where shear studs are more likely to be damaged), and displays an ordered set of results (which would reduce the possibility of a false damage). Further work is required to assess the effects of uncertainties and the adoption of more refined models for the moving load.
\end{abstract}

\footnotetext{
*Corresponding author

Email addresses: S.Kasinos@Lboro.ac.uk (Stavros Kasinos), A.Palmeri@Lboro.ac.uk, Dynamics.Structures@Gmail.com (Alessandro Palmeri), M.Lombardo@Lboro.ac.uk (Mariateresa Lombardo)
} 
Keywords:

Composite bridges, Damage detection, Moving load problem, Structural health monitoring, Non-destructive evaluation

1

\section{Introduction}

Composite steel-concrete beams are widely used in structural engineering, offering the advantages of construction efficiency, durability and improved economy [1-3]. Their performance is strongly influenced by the flexibility of the connection between concrete slab and steel, which generally allows a partial interaction between the two materials. In bridge engineering applications, faster trains and augmented traffic have significantly increased the number and amplitude of loading cycles experienced on a daily basis by composite bridges. This higher demand accelerates the occurrence of damage in the shear connectors, which in turn affects the overall integrity of the structure.

Conventional approaches of damage detection (including ultrasonic, thermal, eddy current and X-ray testing) were termed as cumbersome and expensive, and their application is often limited to the evaluation of local structural performance [4], while visual inspections represent an unreliable solution [5] (also because shear connectors are often inaccessible). Vibration-based damage detection methods have therefore emerged, as they allow identifying meaningful changes in the dynamic characteristics of the composite beam due to alterations in the mechanical properties of the structure [6], with little or no need for the user to know a priori where the damage might be located. Accelerometers have been extensively employed for this purpose, 
although their application to large structural systems like composite bridges may be difficult because of long cabling, number of sensors and installation time. Laser doppler vibrometers (LDVs) can be used as a viable non-contact alternative to accelerometers, especially when targets are difficult to access, but large displacements can adversely affect measurements [7] and the simultaneous acquisition of vibration at multiple points would make very expensive the dynamic testing.

In the general framework of structural health monitoring, vibration-based methods can be classified into "model based methods", which iteratively update the numerical model of the structure to match some dynamic characteristics experimentally measured, and "non-model based methods", which directly compare changes in these characteristics, without any numerical model being required [8]. In both cases, various dynamic characteristics can be exploited as damage-sensitive feature (DSF), including: natural frequencies and modal shapes [9]; modal beam curvatures [10]; frequency response function (FRF) [11]; modal flexibilities [12]; modal strain energy [13].

An early review of different methods of damage detection using natural frequencies can be found in Ref. [14]. However it has become apparent that environmental factors affect eigenfrequencies, which can then mask changes due to damage events [15]. It was also argued that damage does not equally affect all modal frequencies $[4,16]$.

Pascual et al. [17] suggested the use of operating deflection shapes (ODSs) for assessing the presence of damage, while Limongelli [18] proposed an interpolation damage detection method (IDDM), in which the deviation of the deformed shape from a smooth behaviour is used as DSF. Zhang et al. [19] 
proposed the global filtering method (GFM) as detection algorithm for beamand plate-like structures, using ODS curvatures extracted from the dynamic response to moving loads.

When compared to other structural and mechanical systems, limited attempts have been made to apply damage detection methods to shear connectors in composite bridges, with the implementation of vibration-based methods being further restricted by modelling uncertainties of the connectors and low sensitivities. Queiroz et al. [1] investigated full and partial shear connections using nonlinear springs in the FE (finite element) model of composite beams, demonstrating that partial interaction effects should be considered in the analysis. Xia et al. [8] introduced a local identification approach based on the vertical vibration of slab and girders, which does not require baseline data. Dilena and Morassi [20-22] proposed an Euler-Bernoulli beam model to describe the dynamic response of damaged composite beams based on frequency shifts, showing that damage at interior connectors tends to cause lower variations in the modal frequencies, while Liu and De Roeck [23] performed a parametric study, investigating the behaviour of shear connectors during train passages. It was shown that train speed influences the global behaviour of the bridge, and that the longitudinal shear force are not uniformly distributed along the span, with critical regions located near the supports.

While all the above studies use the dynamic response in terms of accelerations and/or displacements at a few locations (analysed in the time domain and/or in the frequency domain), a radically different approach of damage detection and quantification is envisaged in the present research, which consists of analysing the envelope's profile of vehicle-induced deflections in 


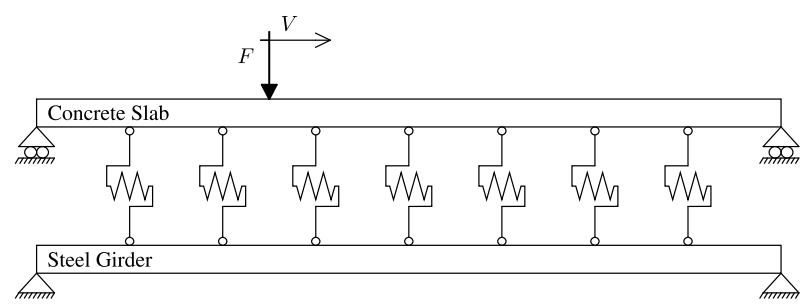

Figure 1: Sketch of the structural problem.

the composite bridge. Instead of considering the whole time history of the dynamic response (and/or its frequency-domain counterpart), the proposed approach only uses the maximum and minimum values of displacements and rotations. Coupled with recent advances in the field of digital image analysis and processing (e.g. deblurring techniques for long-exposure imageries, recently developed by McCarthy et al. [24, 25] for structural dynamics applications), this can lead to an alternative non-contact high-sensitivity method of structural health monitoring for composite bridges, capable of assessing at an early stage the presence and severity of damage.

A set of encouraging preliminary results are presented in this paper, proving the concept in the simple case of a single moving force, although further investigation will be required to assess the effects of uncertainties in the dynamic problem (e.g. random stiffness and random damping of the track $[26,27])$ and to extend this approach to more advanced models for the moving load (e.g. moving masses and moving oscillators [28, 29]).

\section{Envelope-based damage measure}

Let us consider the vehicle-induced vibration of a composite steel-concrete bridge, whose sketch is shown within Figure 1. If a set of moving forces 
is adopted to represent the dynamic load and the structure is assumed to respond within the linear range, the equations of motion for the FE model can be written as:

$$
\mathbf{M} \cdot \ddot{\mathbf{u}}(t)+\mathbf{C} \cdot \dot{\mathbf{u}}(t)+\mathbf{K} \cdot \mathbf{u}(t)=\mathbf{g}+\mathbf{f}(t),
$$

where $\mathbf{u}(t)$ is the array collecting the DoFs (degrees of freedom) of the model; $\mathbf{M}, \mathbf{C}$ and $\mathbf{K}$ are the matrices of mass, equivalent viscous damping and elastic stiffness; while $\mathbf{g}$ and $\mathbf{f}(t)$ are the load vectors associated with the dead and moving forces, respectively. Interestingly, $\mathbf{f}(t)$ depends on the time $t$ not because the magnitude of the applied forces varies, but because they move along the bridge. It is worth mentioning here that, for the sake of simplicity, Eq. (1) does not include the inertia effects due to the moving mass and any vehicle-bridge dynamic interaction phenomena, as they would require timedependent mass, stiffness and damping coefficients [30, 31]. Such refinements of the model would be outside the scope of this work, which is aimed at assessing whether the envelope of the deformations caused by a moving load is sensitive enough to be used in a damage identification scheme instead of changes in the modal frequencies.

Once the governing equations are numerically integrated, the dynamic response of the bridge in terms of displacements and rotations can be expressed as linear combination of the DoFs:

$$
\theta(t)=\mathbf{a}_{\theta}^{\top} \cdot \mathbf{u}(t)
$$

where $\theta(t)$ is the generic response parameter (e.g. deflection at midspan, slope at the supports or the curvature at a given position along the bridge); 


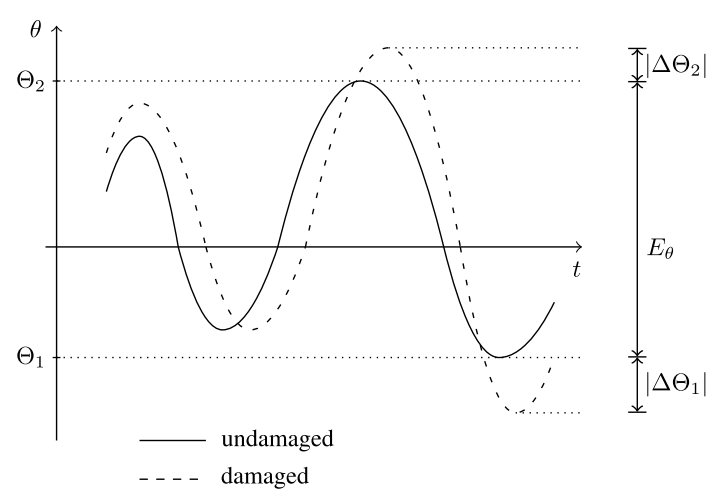

(a)

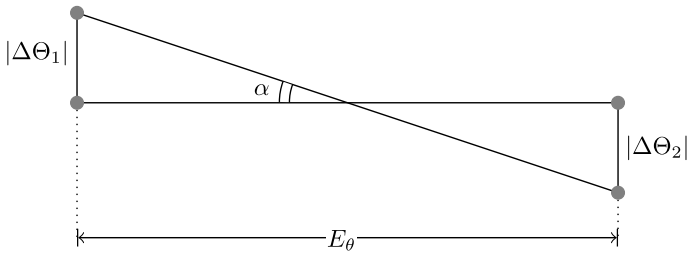

(b)

Figure 2: Modification of the envelope $E_{\theta}$ in a damaged structure (a) and geometrical representation of the damage measure $D_{\theta}=\tan (\alpha)(\mathrm{b})$

$\mathbf{a}_{\theta}$ is the array collecting the associated influence coefficients; and the superscripted symbol $T$ stands for the transpose operator.

It is now possible to introduce the envelope of the dynamic response $\theta(t)$ as the interval $\left[\Theta_{1}, \Theta_{2}\right]$ defined by its extreme values within the selected observation time interval $[0, T]$ :

$$
\Theta_{1}=\min _{0 \leq t \leq T}\{\theta(t)\} ; \Theta_{2}=\max _{0 \leq t \leq T}\{\theta(t)\}
$$

such that $\Theta_{1} \leq \theta(t) \leq \Theta_{2}$ for $0 \leq t \leq T$, and the amplitude of the envelope is (see Figure 2(a)):

$$
E_{\theta}=\Theta_{2}-\Theta_{1}
$$

Alternatively, the amplitude of the envelope can be evaluated as:

$$
E_{\theta}=\left(A_{\theta}^{(+)}+A_{\theta}^{(-)}\right) \theta_{f} .
$$

where $A_{\theta}^{(+)}$and $A_{\theta}^{(-)}$are the dynamic amplification coefficients for the re- 
120

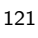

121

sponse parameter $\theta(t)$, given by:

$$
A_{\theta}^{(+)}=\max \left\{\frac{\theta(t)-\theta_{g}}{\theta_{f}}\right\} ; \quad A_{\theta}^{(-)}=\max \left\{-\frac{\theta(t)-\theta_{g}}{\theta_{f}}\right\} ;
$$

$\theta_{g}$ is the static response due to the dead load:

$$
\theta_{g}=\mathbf{a}_{\theta}^{\top} \cdot \mathbf{K}^{-1} \cdot \mathbf{g}
$$

and $\theta_{f}$ if the reference value of the static response due to the moving load, i.e. the largest response obtained when the moving forces are applied statically at different positions on the bridge; formally:

$$
\theta_{f}= \begin{cases}\theta_{f}^{(+)} & \text {if }\left|\theta_{f}^{(+)}\right| \geq\left|\theta_{f}^{(-)}\right| \\ \theta_{f}^{(-)} & \text {otherwise }\end{cases}
$$

where $\theta_{f}^{(+)}=\max \left\{\theta_{i}\right\}$ and $\theta_{f}^{(-)}=\min \left\{\theta_{i}\right\}$ are the maximum and minimum values of the static responses $\theta_{i}=\mathbf{a}_{\theta}^{\top} \cdot \mathbf{K}^{-1} \cdot \mathbf{f}\left(t_{i}\right)$, ideally obtained by freezing the dynamic load vector at different time instants $t=t_{i}$, with $0 \leq t_{i} \leq T$.

If damage occurs, the dynamic response changes and in general the extremes values defining the envelope will be different; that is: $\widetilde{\Theta}_{1} \neq \Theta_{1}$ and $\widetilde{\Theta}_{2} \neq \Theta_{2}$ (in which the over-tilde denotes the quantities affected by the damage).

A dimensionless damage measure $(\mathrm{DM}) D_{\theta}$ can therefore be introduced as:

$$
D_{\theta}=\frac{\left|\Delta \Theta_{1}\right|+\left|\Delta \Theta_{2}\right|}{E_{\theta}},
$$

in which the variation in the extremes of the envelope are:

$$
\Delta \Theta_{1}=\widetilde{\Theta}_{1}-\Theta_{1} ; \quad \Delta \Theta_{2}=\widetilde{\Theta}_{2}-\Theta_{2} .
$$


Figure 2(b) shows that the DM of Eq. (9) can be graphically interpreted as the tangent of the angle $\alpha$ formed by the amplitude of the envelope $E_{\theta}$ on the horizontal axis and the variations of the extreme values $\Delta \Theta_{1}$ and $\Delta \Theta_{2}$ on the vertical axis, that is: $D_{\theta}=\tan (\alpha)$.

For comparison purposes, a more traditional DM can be adopted, based on the reduction in the modal frequencies of the structure. Let $\omega_{1}, \omega_{2}, \cdots$, $\omega_{m}$ be the first $m$ undamped modal circular frequencies of the undamaged composite bridge, solution of the classical real-value eigenproblem $\omega_{i}^{2} \mathbf{M} \cdot \phi_{i}=$ $\mathbf{K} \cdot \phi_{i}$, ordered from the lowest to the highest; and let $\widetilde{\omega}_{1}, \widetilde{\omega}_{2}, \cdots, \widetilde{\omega}_{m}$ be the corresponding frequencies in a given damage scenario. The dimensionless DM associated with the $i$ th modal frequency can be defined as:

$$
S_{i}=\frac{\omega_{i}-\widetilde{\omega}_{i}}{\omega_{i}} .
$$

Depending on the dynamic characteristics of the structure, as well as on location and type of damage, different modal frequencies are differently affected by the damage. For this reason it is worth considering an overall DM for the first $m$ modal frequencies which can be realistically determined with a dynamic test on the composite bridge:

$$
\bar{S}_{m}=\max \left\{S_{1}, S_{2}, \cdots, S_{m}\right\} .
$$

\section{Numerical investigations}

In order to assess the potential for the proposed envelope-based measure $D_{\theta}$ (see Eqs. (9) and (10)) to be used as DSF in civil engineering structures, and specifically in composite steel-concrete bridges, a parametric study has 


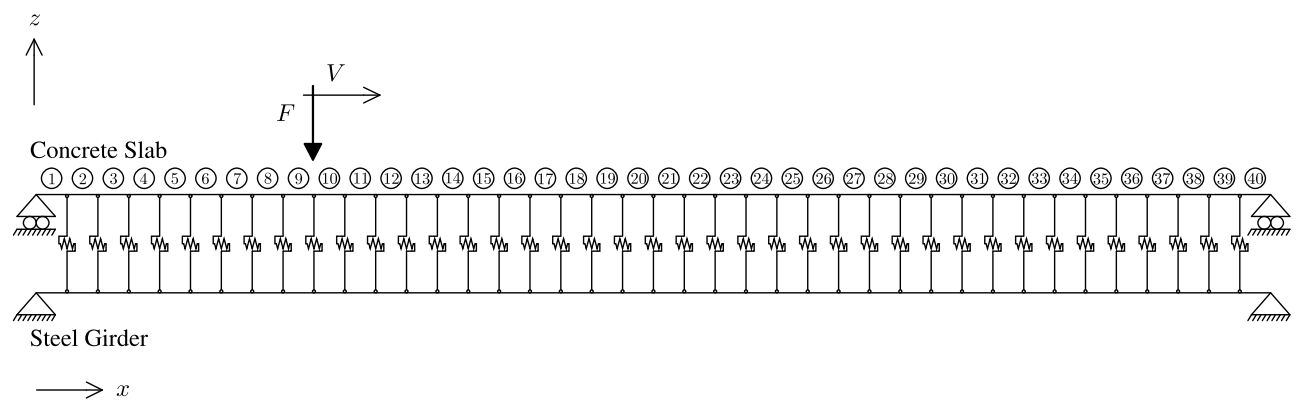

Figure 3: FE model of the composite bridge used as test structure.

been carried out with the idealised FE model of Figure 3, created and validated with the commercial software SAP2000 [32].

Based on an existing structure [6], a single-span simply-supported composite bridge of length $L_{\mathrm{b}}=50 \mathrm{~m}$ has been analysed; the mass density is $\rho_{\mathrm{s}}=7,850 \mathrm{~kg} / \mathrm{m}^{3}$ for the steel and $\rho_{\mathrm{c}}=2,500 \mathrm{~kg} / \mathrm{m}^{3}$ for the concrete; $E_{\mathrm{s}}=206 \mathrm{GPa}$ and $E_{\mathrm{c}}=31 \mathrm{GPa}$ are the corresponding Young's moduli; three values of elastic stiffness have been considered for the shear-type connection between steel and concrete, namely $K_{\mathrm{i}}=0.077 \mathrm{GPa}$ for "soft" interaction and $K_{\mathrm{i}}=0.77 \mathrm{GPa}, K_{\mathrm{i}}=7.7 \mathrm{GPa}$ for "medium" and "stiff" interaction respectively; cross sectional areas, $A_{\mathrm{s}}=7.7 \mathrm{~m}^{2}$ and $A_{\mathrm{c}}=5.6 \mathrm{~m}^{2}$, and second moments, $I_{\mathrm{s}}=11.95 \mathrm{~m}^{4}$ and $I_{\mathrm{c}}=0.0747 \mathrm{~m}^{4}$, fully define the geometry of steel girder and concrete slab, respectively; $d_{\mathrm{b}}=1.5 \mathrm{~m}$ is the distance between the centroids of the two components.

A single concentrated force $F=10 \mathrm{kN}$ has been used as test load, representing a vehicular movement from left to right, with velocities $V=250$ and $300 \mathrm{~km} / \mathrm{h}$ (see Figure 3).

The planar FE model of the objective structure has 201 DoFs and consists of two parallel chords, each one discretised with $N=40$ beam elements, plus 
$N-1$ elastic springs for the shear connectors (which are assumed to be uniformly distributed), while a rigid constraint is applied to the transverse movement of the two chords. As a result, concrete slab and steel girder experience the same amount of deflection but different axial displacements, and the interlayer slip depends on the stiffness of the shear connectors (e.g. $[33,34])$.

\subsection{Dynamic amplification}

In a first stage, the dynamic amplification has been computed for increasing values of the velocity $V$ of the moving force. Figure 4 confirms that the dynamic response of the bridge tends to increase with $V$, for both midspan deflection (denoted with $\delta_{\mathrm{M}}$ ) and rotation at the right end (denoted with $\left.\varphi_{\mathrm{R}}\right)$. In each graph, the top curves $A^{(+)}$refer to movements with the same sign as the corresponding static quantities (i.e. downward displacements at midspan and counterclockwise rotation at the right support of the bridge), while the bottom curves $A^{(-)}$refer to the maxima with opposite sign. Although the pair of $A^{(+)}$and $A^{(-)}$has a very similar trend in the two graphs, there are some differences, e.g. the right rotation tends to show higher values of dynamic amplification for $V>400 \mathrm{~km} \mathrm{~h}^{-1}$, while relative maxima of the dynamic amplification can occur at different velocities, e.g. $V=280 \mathrm{~km} \mathrm{~h}^{-1}$ for $A_{\delta_{\mathrm{M}}}^{(+)}$and $V=310 \mathrm{~km} \mathrm{~h}^{-1}$ for $A_{\varphi_{\mathrm{R}}}^{(+)}$. Interestingly, the dynamic amplification is also seen to increase with the flexibility of the connection, particularly at higher values of $V$.

Figure 5 shows the envelopes $E_{\delta_{\mathrm{M}}}$ and $E_{\varphi_{\mathrm{R}}}$, normalised with respect to the corresponding reference values of the static response (see Eq. (5)). It appears that the envelope is highly sensitive to the velocity $V$ of the moving force. 


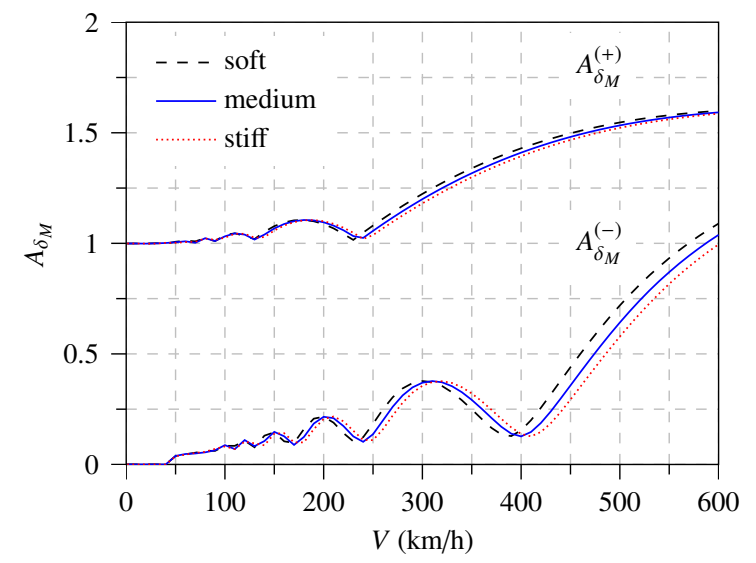

(a)

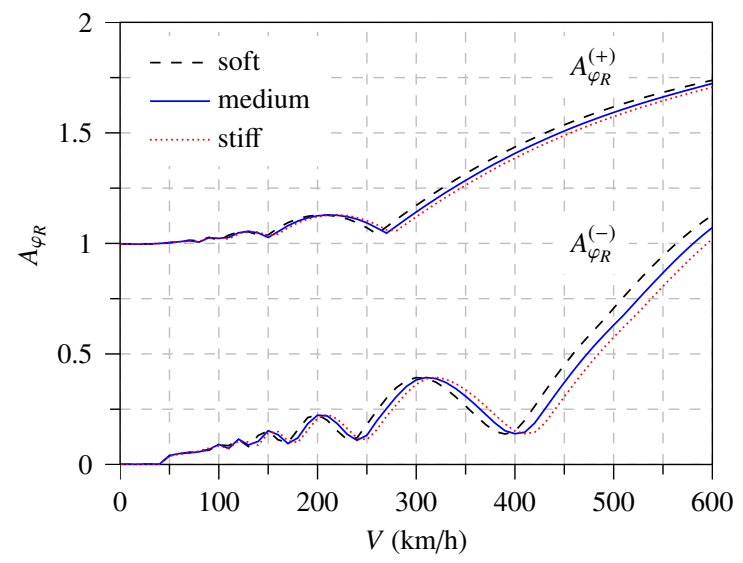

(b)

Figure 4: Dynamic amplification factors for (a) midspan displacement, (b) right support rotation

In both graphs, for instance, a relative valley and a relative peak appear for velocities close to $V=250 \mathrm{~km} / \mathrm{h}$ and $V=300 \mathrm{~km} / \mathrm{h}$, and these values have therefore been used in the next set of dynamic analyses with moving forces.

\subsection{Damage sensitivity}

\subsubsection{Modal frequencies}

Stiffness reduction at a given location of the structure generally causes the modal frequencies to drop, which in turn can indicate the presence of damage at a global level. However the same amount of damage at different locations may induce different amount of frequency changes. A parametric study has then been carried out to quantify the effectiveness of such variations as detection feature of a damage occurring at the interface between concrete slab and steel girder in the objective composite bridge. Figure 6 shows the colour maps of the sensitivity matrices $\mathbf{S}$ for two values of the elastic stiffness 


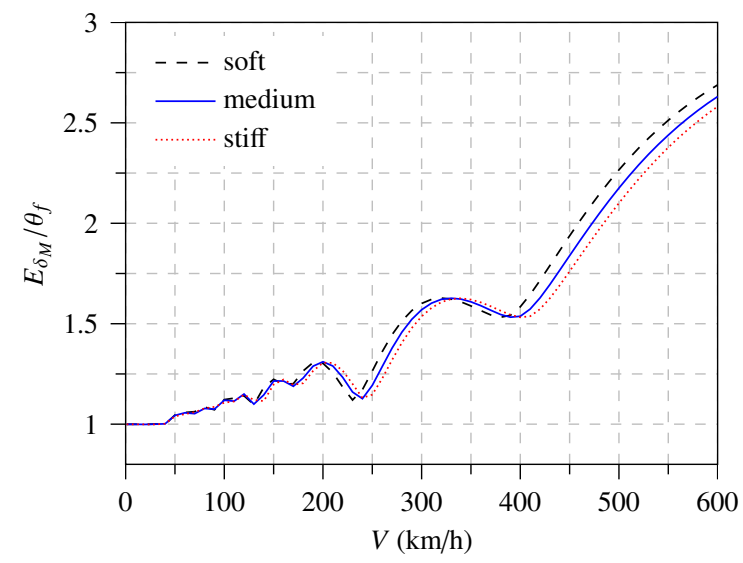

(a)

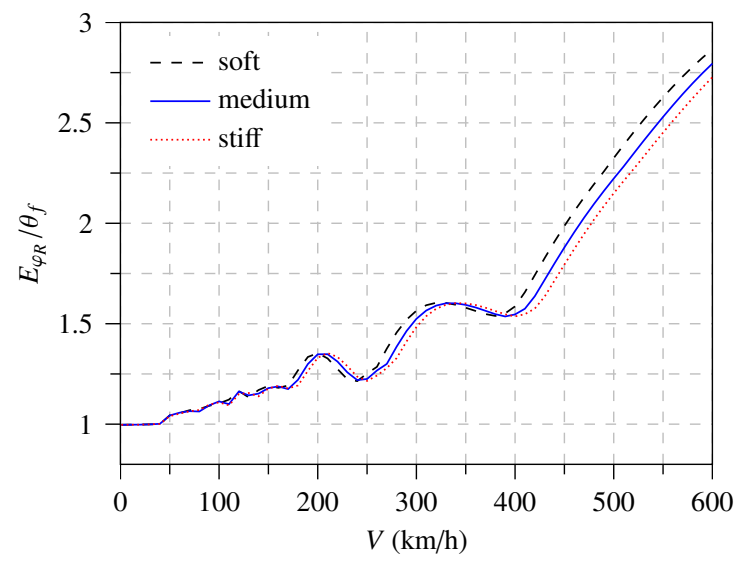

(b)

Figure 5: Envelope of dynamic amplification factors for (a) midspan displacement, (b) right support rotation

of the shear connectors, assuming in both cases that the localised damage corresponds in the FE model to a $90 \%$ reduction in the stiffness of the $j$ th shear spring. The generic coefficient $f_{i, j}$ of each matrix is the dimensionless frequency shift $S_{i}$ of Eq. (11) for a damage occurring at the $j$ th position $x_{j}=j \Delta x$, in which $j=1, \cdots, N-1$ and $\Delta x=L_{\mathrm{b}} / N=125 \mathrm{~cm}$ is the size of the FE discretisation.

The two colour maps lend themselves to the following considerations:

1. Due to the symmetry of the problem, the maps are symmetric with respect to the midspan position $(j=20=N / 2)$;

2. The sensitivity tends to increase with the level of partial interaction;

3. For each mode $i$, the sensitivity is higher when the location $x_{j}$ of the damage is close to a point of contraflexure in the associated modal shape, e.g. close to the ends of the bridge (i.e. $j=1$ and $j=N-1$ ), where the shear force is larger; 


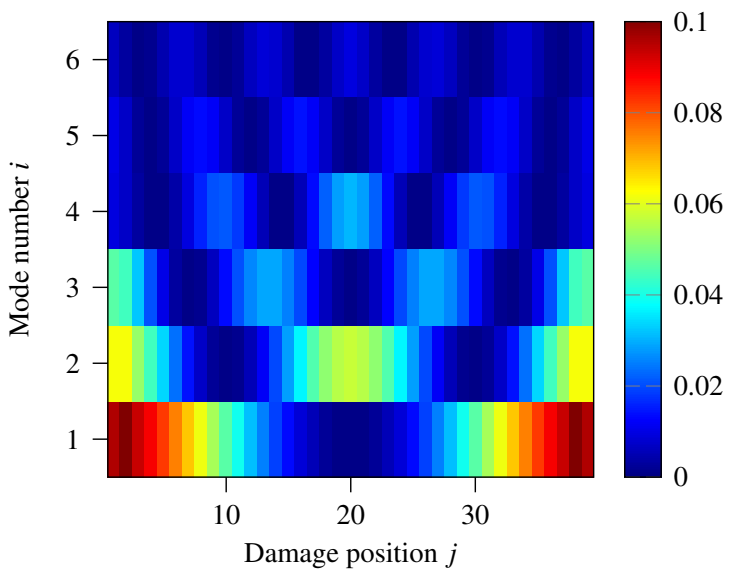

(a)

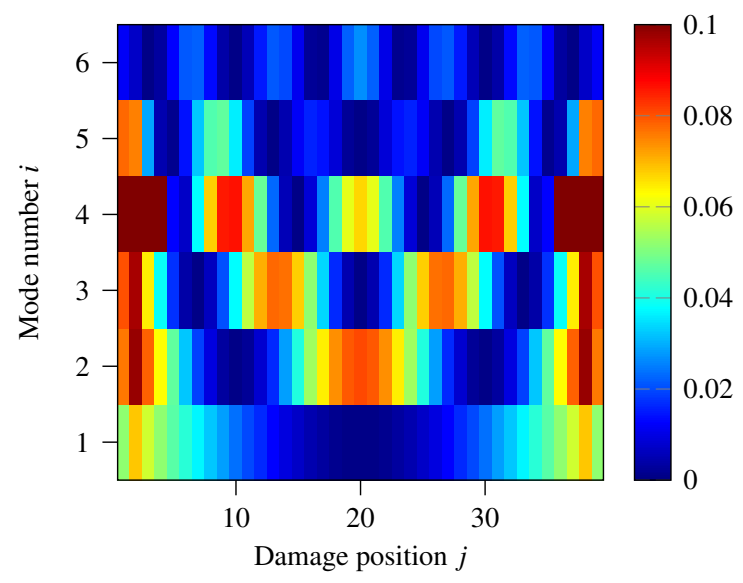

(b)

Figure 6: Damage sensitivities $f_{i, j}$ for the natural frequencies associated with the first eight flexural modes of vibration in case of medium (a) and stiff (b) partial interaction.

4. Conversely, the sensitivity of a given mode $i$ reduces when the location $x_{j}$ of the damage is close to a zero-value point in the shear force diagram of the associated modal shape (that is, if the shear force is relatively small, the effect of a damage in the shear studs at that position will be relatively negligible).

As a consequence, the first mode of vibration only shows a good level of sensitivity if the damage occurs near to the supports of the bridge, while higher modes of vibration reveal damage at additional positions. Furthermore, different modes have different sensitivity levels for the same damage position (e.g. a damage at midspan affects second and fourth mode of vibration, as shown by a warm spot in the colour maps, but does not affect first and third mode). Therefore, different modes of vibration are required to detect the presence of damage, meaning that a large number of sensors 
may be required for practical applications.

A further observation is that the mode number $i$ with the highest sensitivity $S_{i}$ to damage in the shear connectors at $x=x_{j}$ may vary with the level of partial interaction between the concrete slab and shell girder. Indeed, while for the case of medium interaction (Figure 6(a)) the first mode shows the highest sensitivity values for position index $j \leq 5$ (and $j \geq 35$ ), modes $i=2,3$ and 4 appear to be more sensitive in the case of stiff interaction (Figure 6(b)).

\subsubsection{Envelope of deflections}

In a second stage, it has been numerically verified that the envelope $E_{\delta_{i}}$ of the deflection $\delta_{i}(t)$ at the output position $x=x_{i-1}$ can be used as sensitive feature for a localised damage in the shear connector at the position $x=x_{j}$, with $1 \leq i \leq N+1$ and $1 \leq j \leq N-1$. Considering all the possible combinations of output position and damage position in the FE model, the relevant sensitivity matrix $\mathbf{D}$ has been obtained, where the generic element $d_{i, j}$ is the dimensionless DM $D_{\delta_{i}}$ of Eq. (9), in which: $\theta(t)=\delta_{i}(t)$; damage occurs at the $j$ th shear spring; the observation time interval is $\left[0, L_{b} / V\right]$, which corresponds to the time required by the moving force $F$ to cross the bridge.

A set of $N-1$ time-history analyses was therefore required (i.e. one analysis for each damage location), and this was repeated four times (as two levels of partial interaction $K_{\mathrm{i}}$, medium and stiff, and two velocities of the moving force $V$ were studied). Including the undamaged scenarios, a total of 158 dynamic analyses were carried out, whose results are summarised with the four colour maps of Figure 7, in which warmer colours show where the 
sensitivity to the damage is higher.

In comparison with the results of Figure 6, higher values of sensitivity have been computed, meaning that the envelope of displacements is potentially more effective than the modal frequency shift as DSF (that is, the maximum frequency sensitivity $f_{i, j}$ in Figure 6 is about 0.1 , while the sensitivity of $E_{\delta_{i}}$ in Figure 7 is about 0.6, more than five times higher). Clearly the actual performance of the method will depend on the velocity $V$ of the moving force, which therefore needs to be carefully selected. For instance, at relatively low value of $V$, say, $V<100 \mathrm{~km} / \mathrm{h}$ in the case study, very little dynamic effects are expected, and therefore any attempt to identify the presence of damage in the bridge could become difficult for the presence of noise in the measurements and other forms of uncertainties.

Additionally, the sensitivity coefficients $d_{i, j}$ are higher at the ends of the bridge, i.e. for $j \leq 5$ or $j \geq 35$, and tend to decrease with the distance between damage position and output position, i.e. with $|i-j|$. While the first feature is acceptable from an engineering point of view, since damage in shear studs is unlikely to happen toward the middle of the bridge, where lower levels of shear stress are expected, the second feature is highly desirable, as it makes easier the localisation of the damage by looking at the position where the maximum variation in the envelope of displacements is observed.

Interestingly, the effects of damage on the envelope $E_{\delta_{i}}$ are more localised in the case of stiff concrete-steel interaction, while comparatively the variation in the velocity $V$ has a less significant impact on the sensitivity coefficients $d_{i, j}$. 


\subsubsection{Envelope of rotations and curvatures}

Further sensitivity analyses were carried out on the test bridge using the rotation $\varphi_{i}$ and the curvature $\chi_{i}$ at the generic abscissa $x=x_{i-1}$ as DSFs (with $i=1, \cdots, N+1$ ). While the rotation $\varphi_{i}$ was obtained directly from the dynamic analyses (being a DoF of the FE model), the curvature was computed as $\chi_{i}=M_{s}\left(x_{i-1}\right) / E_{s} I_{s}, M_{s}(x)$ being the bending moment in the steel girder at the generic abscissa $x$. The results in terms of rotation's sensitivity coefficients $r_{i, j}$ and curvature's sensitivity coefficients $q_{i, j}$ are presented in Figures 8 and 9, respectively, being $r_{i, j}=D_{\varphi_{i}}$ and $q_{i, j}=D_{\chi_{i}}$ for a concentrated damage occurring at the $j$ th shear spring in the FE model.

The same trends predicted by the envelope of displacements are verified for the case of rotations. In particular, similar sensitivity levels have been computed for the rotations at the ends of the bridge, and the localisation tends to improve with the rigidity of the inner layer. Interestingly, a rotations' sensitivity to damage increases at midspan position with respect to the envelope of the displacements.

The results for the envelope of the curvatures are quite different. In particular, their sensitivity shows large peaks closer to the ends of the bridge, while reduced values are noted elsewhere. Moreover, increased sensitivity is also observed for the stiffer shear connectors, with minimal differences due to the velocity of the load.

\subsubsection{Comparison}

In order to assess the relative performance of different DSFs for the composite bridge under consideration, the maximum value attained by the various sensitivity coefficients has been computed for each damage position $j$, e.g. 
$f_{j}=\max \left\{f_{i, j}, i \leq 6\right\}$ for the frequency shifts $d_{j}=\max \left\{d_{i, j}, i \leq N+1\right\}$ for the envelope of displacements. The semi-logarithmic plots of Figure 10 compare the four DSFs $f_{j}, d_{j}, r_{j}$ (envelope of rotations) and $q_{j}$ (envelope of curvatures) for two velocities of the moving load and two levels of partial steel-concrete interaction. It appears that the curvature (dotted blue lines) is highly sensitive to damage occurring close to the ends of the bridge. Unfortunately, it is particularly difficult to track curvature changes using non-contact measurements on a bridge structure, and for this reason the envelope of the curvature appears as the least practical approach.

Shifts in the natural frequencies (green solid lines) are very effective for stiff partial interaction and damage close to the ends of the bridge (see Figures 10(b) and (d)). However, a sudden drop follows when moving towards the middle of the bridge. Importantly, while this DSF is independent of the load velocity, as it only uses modal information, its performance is highly dependent on the level of partial interaction, and indeed for the case of medium stiffness this is less effective approach (see Figures 10(a) and (c)).

The envelope of both displacements (red dashed lines) and rotations (black dot-dashed lines) appear as viable DSFs, with on average a slightly better performance for the rotations, although in practical applications it would be easier to get the displacements. It must also be stressed that, if an imagery type of approach is used to determine the envelopes (e.g. Refs. [24] and [25]), deflection and rotations can potentially be simultaneously tracked. It is also worth stressing here that in practical applications the envelope of both the undamaged and damaged bridge must be available under the same loading conditions, as correlating the two dynamic responses will allow 
identifying the damage.

\section{Conclusions}

In this paper, a novel approach for damage detection in composite steelconcrete bridges is suggested, in which the envelope of deflections and rotations induced by moving loads are used as DSFs (damage-sensitive features). While in traditional vibration-based approaches discrete-time signals of displacements, strains or accelerations from field experimentation are collected and analysed (either in the time domain or in the frequency domain), the proposed approach only requires the extreme values of the dynamic response to be known. As hardware and software for digital imagery continuously progress, such information can potentially be acquired more economically and more easily than in conventional methods. Moreover, since no special sensors are needed, but just visible targets, more deflections and rotations can be simultaneously monitored, which can improve the accuracy of damage detection.

To prove the concepts, numerical analyses have been carried out on the finite element model of a realistic composite bridge, assuming a single moving force as dynamic excitation. In a first stage, it has been shown that the dynamic effects associated with the moving load are significant, and tend to increase with the flexibility of the shear connectors between concrete slab and steel girder.

In a second stage, the effects of damage at different locations were quantified for both medium and stiff partial interaction and for two velocities of the moving force. In this way, any significant anomaly in the performance of 
the proposed approach could have been spotted.

As expected, the results have demonstrated that the envelope of the dynamic response in terms of deflections and rotations tends to increase when damage occurs. More importantly, about the same level of sensitivity to damage was observed for shifts in the modal frequencies (which in the current practice is often used as DSF) and variations in the envelope of deflections and rotations, whose sensitivity did not suffer from significant changes when the level of partial interaction and the velocity of the moving force were varied. Additionally, the proposed approach was found to be most effective closer to the ends of the bridge, where damage is more likely to happen, and was shown to display an ordered set of results, that can potentially enhance the predictiveness of any damage-detection algorithm.

Although these preliminary results are very promising, further numerical and experimental investigations need to be undertaken to fully develop the method, explore its practical limitations and verify the application to real structures. Moreover, due to the scalability of the imageries for the extraction of the envelopes, this new approach could be potentially applied to structures at different scales, from large civil-engineering buildings and bridges to mechanical components and even nano-devices.

\section{Acknowledgements}

The present research was conducted as part of the ENSURE scheme of Loughborough University, whose financial contribution is gratefully acknowledged. 


\section{References}

1. Queiroz FD, Vellasco PCGS, Nethercot DA. Finite element modelling of composite beams with full and partial shear connection. Journal of Constructional Steel Research 2007;63:505-21.

2. Ranzi G, Zona A. A steel-concrete composite beam model with partial interaction including the shear deformability of the steel component. Engineering Structures 2007;29:3026-41.

3. Liu K, Reynders E, Roeck GD, Lombaert G. Experimental and numerical analysis of a composite bridge or high-speed trains. Journal of Sound and Vibration 2009;320:201-20.

4. Khoo LM, Mantena PR, Jadhav P. Structural damage assessment using vibration modal analysis. Structural Health Monitoring 2004;3:177-94.

5. Beskhyroun S, Wegner LD, Sparling BF. New methodology for the application of vibration-based damage detection techniques. Journal of Structural Control and Health Monitoring 2012;19:632-49.

6. Liu K, De Roeck G. Damage detection of shear connectors in composite bridges. Structural Health Monitoring 2009;65:1105-11.

7. Warren C, Niezrecki C, Avitabile P, Pingle P. Comparison of FRF measurements and mode shapes determined using optically image based, laser, and accelerometer measurements. Mechanical Systems and Signal Processing 2011;25:2191-202. 
8. Xia Y, Hao H, Deeks AJ. Dynamic assessment of shear connectors in slab-girder bridges. Engineering Structures 2007;29:1475-86.

9. Ndambi JM, Vantomme J, Harri K. Damage assessment in reinforced concrete beams using eigenfrequencies and mode shape derivatives. Engineering Structures 2002;24:501-15.

10. Wahab MM, De Roeck G. Damage detection in bridges using modal curvatures: application to a real damage scenario. Journal of Sound and Vibration 1999;226(2):217-35.

11. Rad S. Methods for updating numerical models in structural dynamics. Phd thesis; 1997.

12. Pandey AK, Biswas M. Damage detection in structures using changes in flexibility. Journal of Sound and Vibration 1994;169:3-17.

13. Shi ZY, Law SS. Structural damage localization from modal strain energy change. Journal of Sound and Vibration 1998;218:825-44.

14. Salawu O. Detection of structural damage through changes in frequency: A review. Engineering Structures 1997;19:718-23.

15. Peeters B, Maeck J, De Roeck G. Vibration-based damage detection in civil engineering: Excitation sources and temperature effects. Smart Materials and Structure 2001;10:518-27.

16. Zhou Z. Vibration-based damage detection of simple bridge superstructures. Phd thesis; 2006. 
17. Pascual R, Golinval J, Razeto M. On-line damage assessment using operating deflection shapes. In: IMAC XVIII: 17th International Modal Analysis Conference. San Antonio (TX), USA; 1999, p. 1225-31.

18. Limongelli MP. Frequency response function interpolation for damage detection under changing environment. Mechanical Systems and Signal Processing 2010;24:2898-913.

19. Zhang Y, Lie S, Xiang Z. Damage detection method based on operating deflection shape curvature extracted from dynamic response of a passing vehicle. Mechanical Systems and Signal Processing 2013;35:238-54.

20. Dilena M, Morassi A. A damage analysis of steel-concrete composite beams via dynamic methods: Part II. analytical models and damage detection. Journal of Vibration and Control 2003;9:529-65.

21. Dilena M, Morassi A. Experimental modal analysis of steel concrete composite beams with partially damaged connection. Journal of Vibration and Control 2004;10(6):897-913.

22. Dilena M, Morassi A. Vibrations of steel-concrete composite beams with partially degraded connection and applications to damage detection. Journal of Sound and Vibration 2009;320:101-24.

23. Liu K, De Roeck G. Parametric study and fatigue-life-cycle design of shear studs in composite bridges. Journal of Constructional Steel Research $2009 ; 8: 345-56$.

24. McCarthy D, Chandler JH, Palmeri A. Monitoring dynamic structural 
tests using image deblurring techniques. Key Engineering Materials 2013;569-570:932-9.

25. McCarthy D, Chandler JH, Palmeri A. 3D case studies of monitoring dynamic structural tests using long exposure imagery. In: ISPRS: The International Archives of the Photogrammetry, Remote Sensing and Spatial Information Sciences; vol. XL-5. 2014, p. 407-11.

26. Muscolino G, Palmeri A, Sofi A. Vibration of slender composite beams with flexible shear connection under moving oscillators. In: ICASP 2010: 10th International Conference on Applications of Statistics and Probability. Tokyo, Japan; 2010, .

27. Bryja D, Hoł ubowski R. Galerkin finite element modelling and monte carlo solutions for a railway bridge with random track ballast. In: CC 2013: 14th International Conference on Civil, Structural and Environmental Engineering Computing,. Cagliari, Italy; 2013, .

28. Biondi B, Muscolino G, Sofi A. A substructure approach for the dynamic analysis of train-track-bridge system. Computer and Structures 2012; 83:2271-81.

29. Stăncioiu D, Ouyang H, Mottershead JE. Vibration of a beam excited by a moving oscillator considering separation and reattachments. Journal of Sound and Vibration 2008;310:1128-40.

30. Muscolino G, Palmeri A, Sofi A. Absolute versus relative formulations of the moving oscillator problem. International Journal of Solids and Structures 2009;46:1085-94. 
471

472

473

31. De Salvo V, Muscolino G, Palmeri A. A substructure approach tailored to the dynamic analysis of multi-span continuous beams under moving loads. Journal of Sound and Vibration 2010;329:3101-20.

32. SAP2000 . Release 15.2.1. Computers and Structures; Berkeley (CA), USA; 2014.

33. Newmark NM, Siess CP, Viest IM. Tests and analysis of composite beams with incomplete interaction. Proceedings of the Society for Experimental Stress Analysis 1951;9:75-42.

34. Palmeri A. Vibration of slender composite beams with flexible shear connection under moving oscillators. In: RASD 2010: 10th International Conference on Recent Advances in Structural Dynamics. Southampton, $\mathrm{UK} ; 2010$, . 


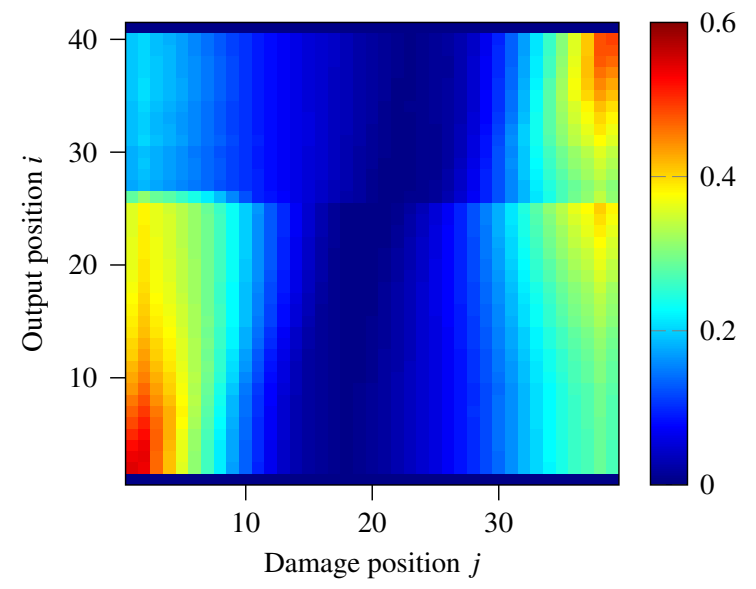

(a)

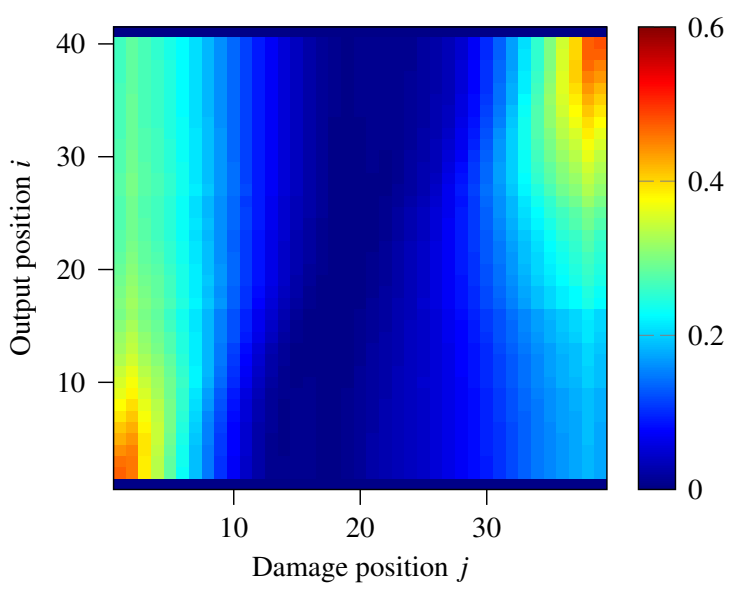

(c)

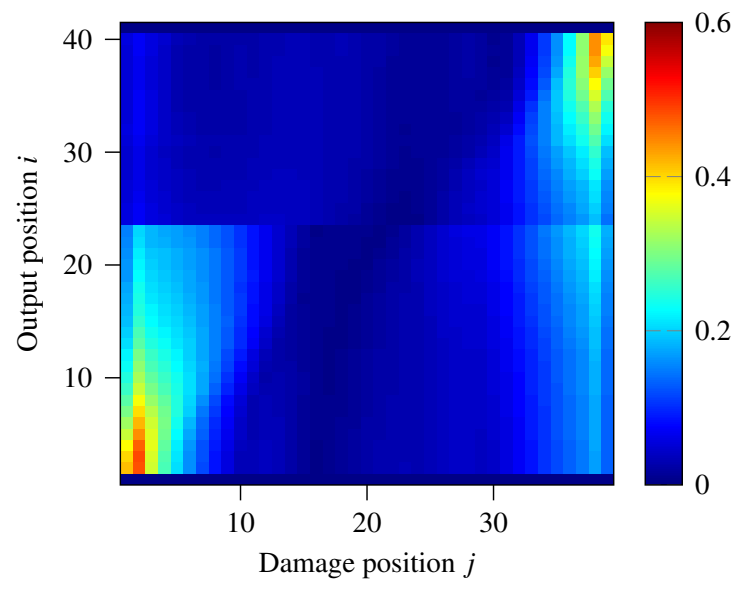

(b)

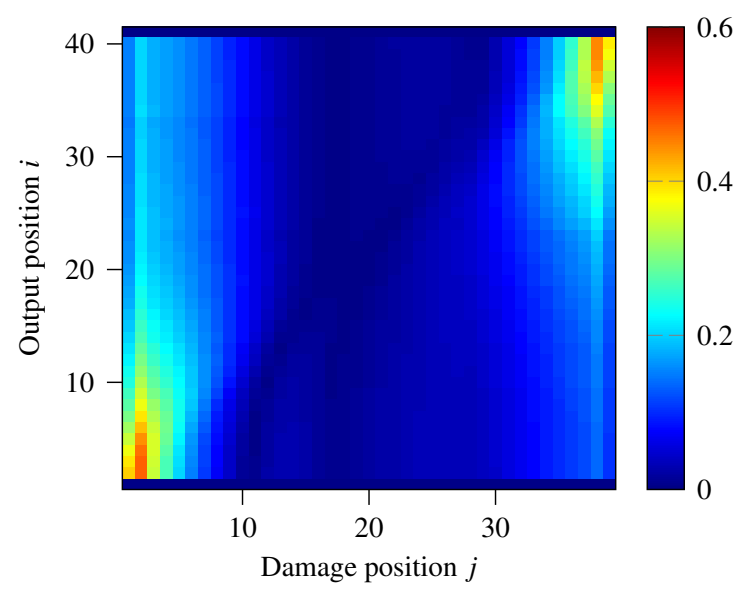

(d)

Figure 7: Damage sensitivities $d_{i, j}$ for the displacement's envelope $E_{\delta_{i}}$ in case of medium (left) and stiff (right) partial interactions at velocities of the force $V=250 \mathrm{~km} / \mathrm{h}$ (top) and $300 \mathrm{~km} / \mathrm{h}$ (bottom). 


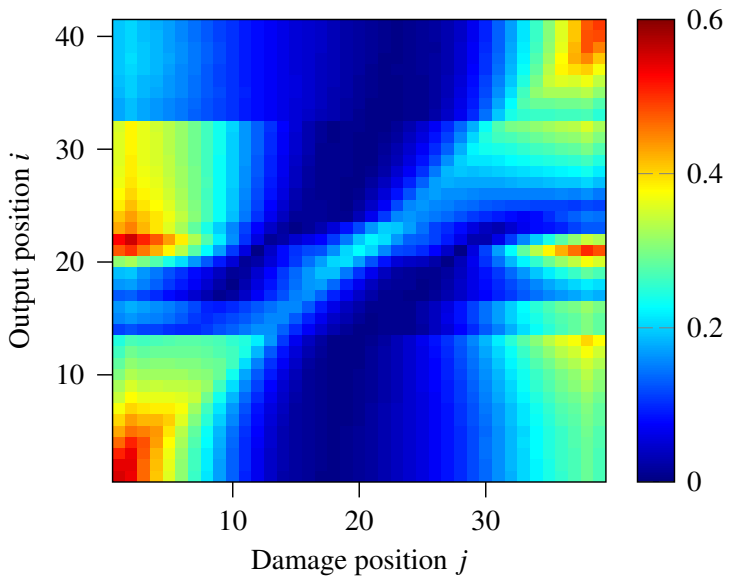

(a)

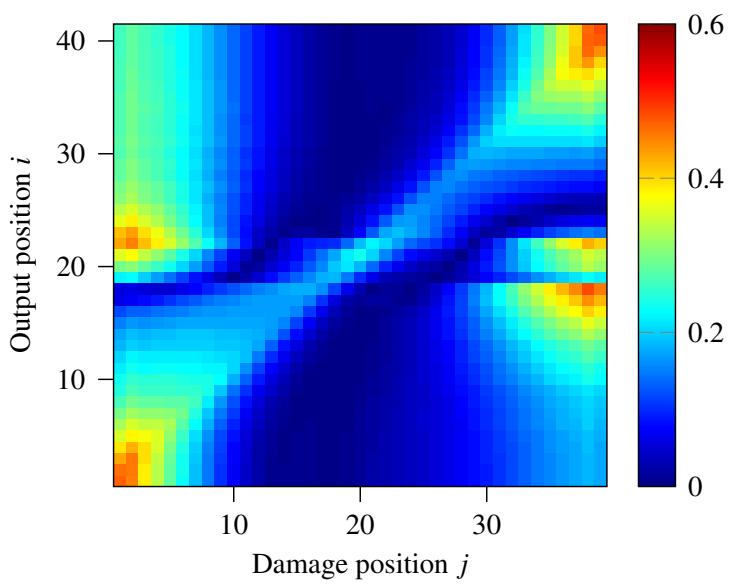

(c)

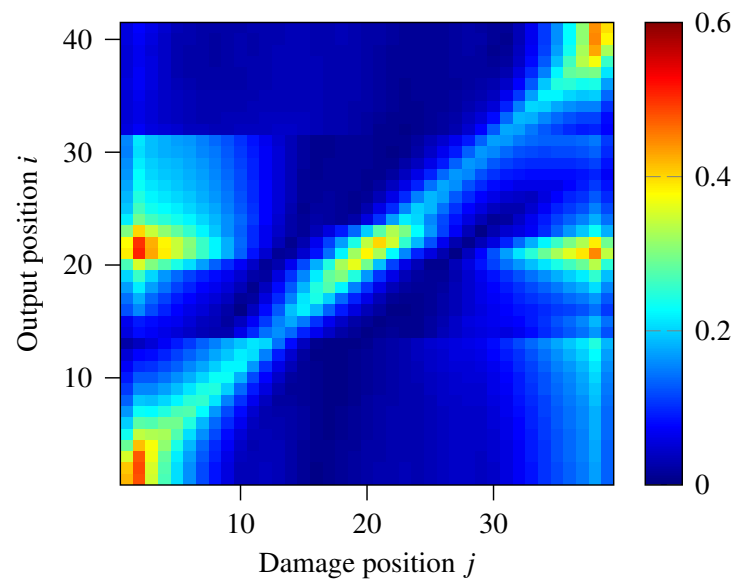

(b)

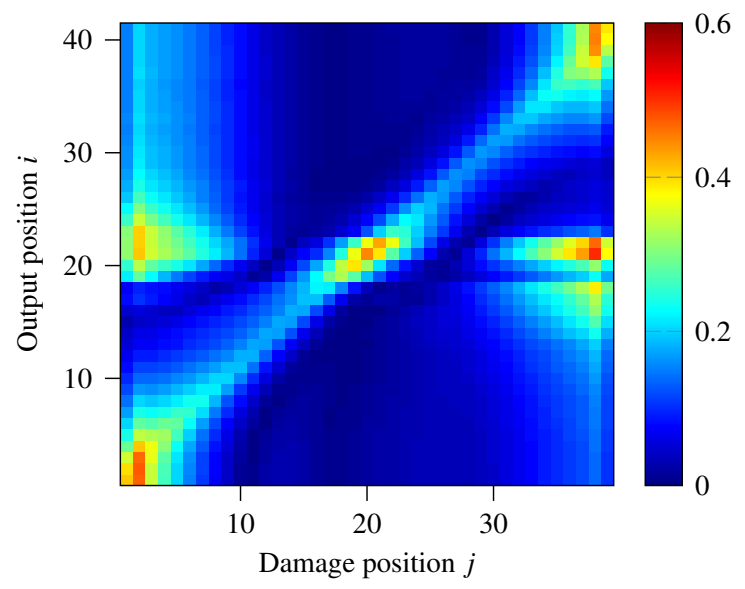

(d)

Figure 8: Damage sensitivities $r_{i, j}$ for the rotation's envelope $E_{\delta_{i}}$ in case of medium (left) and stiff (right) partial interactions at velocities of the force $V=250 \mathrm{~km} / \mathrm{h}$ (top) and $300 \mathrm{~km} / \mathrm{h}$ (bottom). 


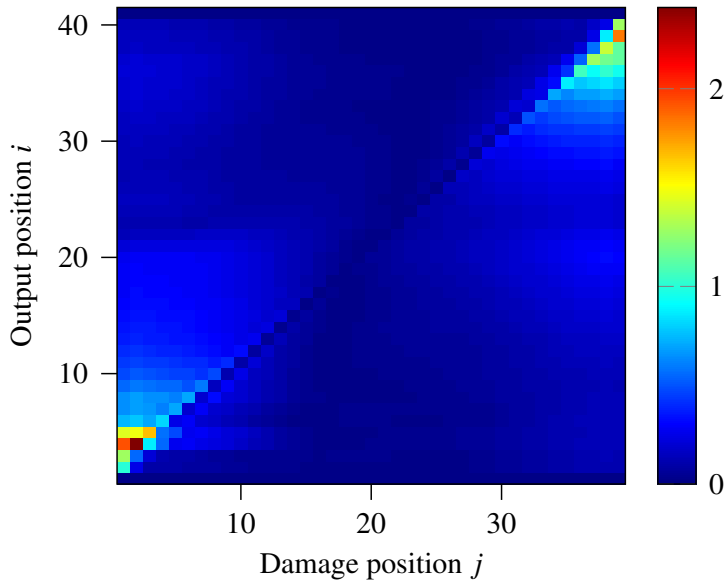

(a)

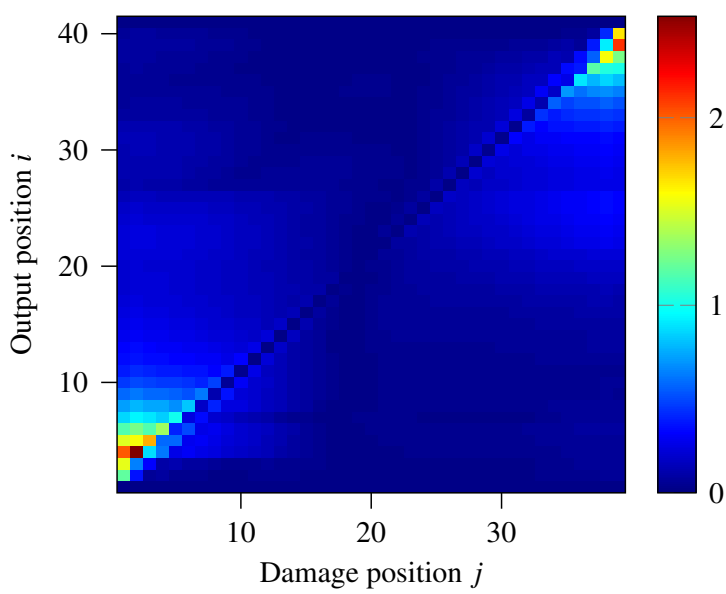

(c)

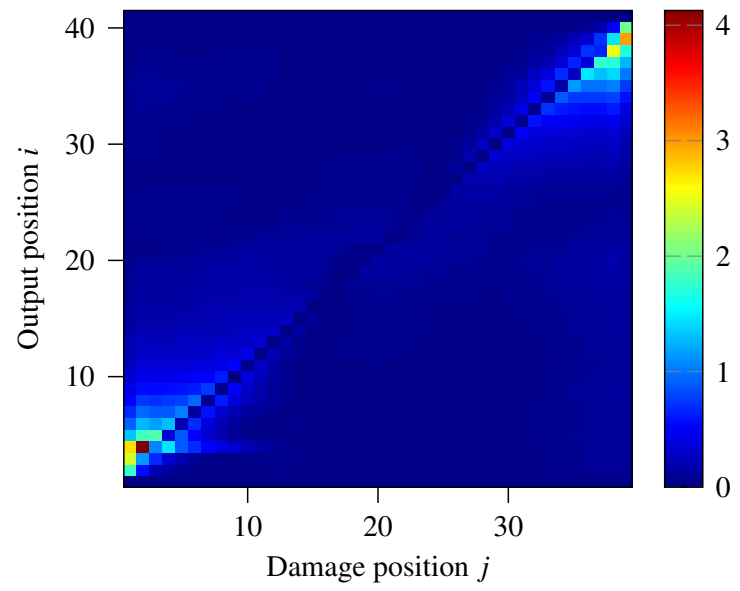

(b)

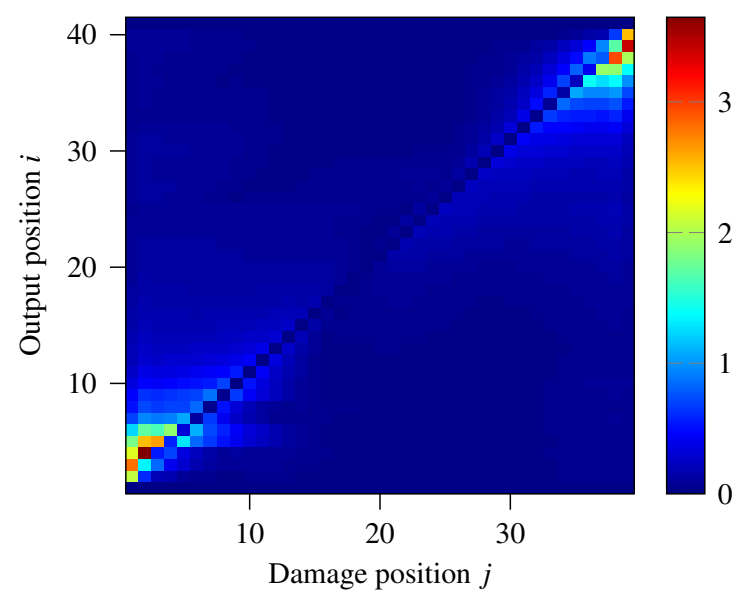

(d)

Figure 9: Damage sensitivities $q_{i, j}$ for the curvature's envelope $E_{\delta_{i}}$ in case of medium (left) and stiff (right) partial interactions at velocities of the force $V=250 \mathrm{~km} / \mathrm{h}$ (top) and $300 \mathrm{~km} / \mathrm{h}$ (bottom). 


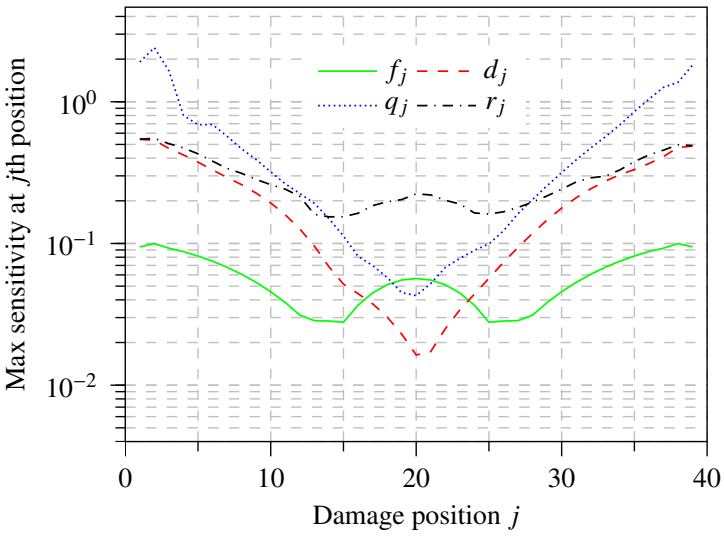

(a)

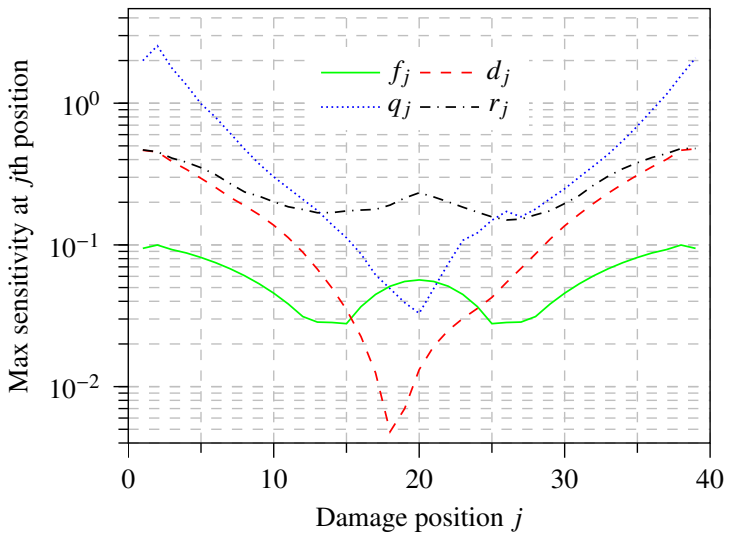

(c)

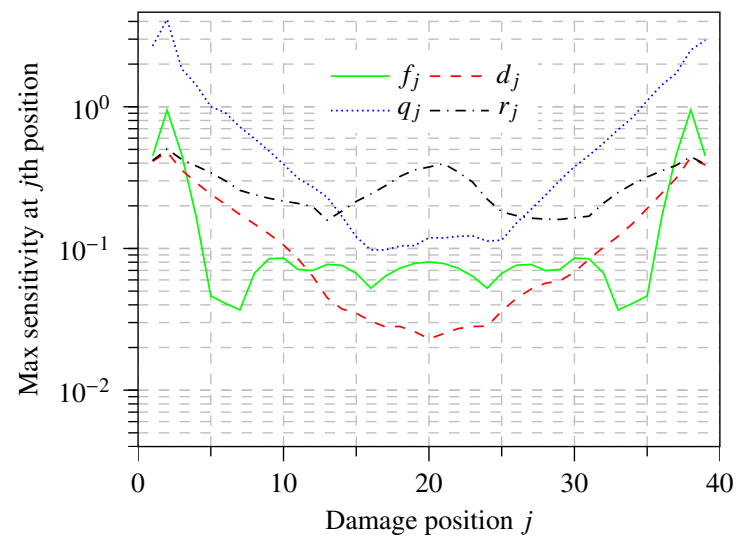

(b)

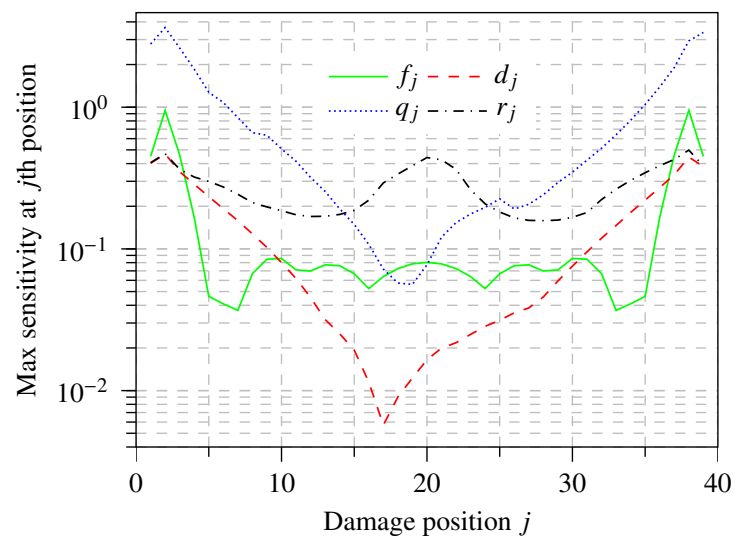

(d)

Figure 10: Performance of different damage-sensitive features in case of medium (left) and stiff (right) partial interactions at velocities of the force $V=250 \mathrm{~km} / \mathrm{h}$ (top) and $300 \mathrm{~km} / \mathrm{h}$ (bottom). 